\title{
Image Qualiy Improvement Using Algorithm Spatial Median Filter And Adaptive Fuzzy Contrast Enhacement
}

\author{
Preddy Marpaung ${ }^{1}$, Muhammad Zarlis ${ }^{1}$ and Zakarias Situmorang ${ }^{1}$ \\ \{preddymarpaungusu2015@gmail.com\} \\ ${ }^{1}$ Faculty of Computer Science and Information Technology, Universitas Sumatera Utara, Indonesia
}

\begin{abstract}
This study aims to increase the image quality in terms of contrast and noise removal, it is necessary to do processing on the image in order to be interpreted in accordance with the desired. Image in question is a static grayscale images with 8-bit PNG that have low contrast and noise levels above $10 \%$ ie $15 \%-50 \%$ at intervals of $5 \%$. The author uses algorithms Median Spatial Filter (SMF) to reduce noise and Fuzzy Adaptive Contrast Enhancement Algorithm With Details Serving (AFCDP) for enhancing the contrast, where the two algorithms are combined. This technique is excellent in image enhancement, where the results of tests performed combination model can improve the image of the highest quality at $15 \%$ the percentage of noise with PSNR $=26.417$ and $\mathrm{SE}=0.0387$.
\end{abstract}

Keywords: Image quality, Image processing, Noise reduction and Contrast enhancement, and a Combination SMF AFCDP.

\section{Introduction}

Improving the quality of the image (Image Enhancement) is an interesting thing with the challenges inherent in image processing, this also applies to the contrast enhancement or image quality improvement [1]. Some of the images may decrease the quality to be darker even damaged can be caused by noise or because of damage to the recording device cause the object in the image becomes unclear, making it difficult to be processed again [2] [3]. Type gaussian noise and impulse noise, better known as salt and pepper noise in the form of black or white dot is the noise that is often found in the image[4][2], In addition the level of contrast of the image also becomes important because it affects the image quality, especially the content of the image, making it lookthe need for a deeper study to increase image quality in terms of contrast and noise removal [3][4][5].

Some of the existing research, apply image enhancement separately between noise removal and contrast enhancement. Kohli and Kaur doing research by comparing the two algorithms eliminate the salt and pepper noise with Spatial Median Filter algorithm (SMF) and Adaptive Noise Reduction, obtained SMF algorithm capable of reducing noise with better outcomes [6].

Rajesh Kumar [6] also conduct research to compare with Spatial Filter Spatial Mean Median Filter with three scalar colors and varying noise levels obtained Spatial Filter Median give better results. Improved image quality in addition to improvements to the salt and pepper 
noise as well as contrast enhancement. Similar research has been done to improve the quality of image contrast by using Fuzzy Adaptive Contrast Enhancement Algorithm With Details Preserving (AFCEDP) compared to using the same algorithms Adaptive Contrast Enhancement Algorithm difference without using fuzzy [6] obtained AFCEDP get better results without significantly reducing image information [6], Increase the contrast by using brightness and details preserving give better results [7]. In addition there are also applying image contrast enhancement with Fuzzy Method are also able to enhance the image contrast nicely compared with the method of fuzzy sets enhancement, NINT, Pal-King, fuzzy-rulebased,fuzzy Quantitive Measure, Local Fuzzy Enhancement [5],

Based on the reference, the combination of SMF algorithm for noise removal salt and pepper and AFCEDP for enhancing the contrast has never been tested, so we need a more indepth study in the hope that more will be found ideal models to cope with noise and low contrast with the quality of the relatively better. Ingeneral, the study was designed as follows: In the first image dataset will be tested in two models, the first one; eliminate noise followed by peningkatanan contrast, second; contrast enhancement first, followed by the removal of noise to get the model better testing by comparing the results of which will be the process of calculating the change in the image of the noise removal using the Mean Signal Error (MSE) and Peak Signal To Noise Ratio (PSNR) and for enhancing the contrast using Shannon Entropy (SE) and Contrast Improvement Evaluation (CIE). To get relatively better results, further testing needs to be done so it can be the best algorithm combination model that measured both in terms of data,

\section{Method}

\subsection{Method of collecting data}

The data used is the data set in the form of pictures PNG 8-Bit 5 pieces with levels of low contrast and the percentage of noise salt above 10\% ie 15\% - 50\% at intervals of 5\% to be tested each with content images used varied without referring to certain things. Here is an example of a low contrast image of the image data and noise above $10 \%$ as varied as shown in Figure 2.1 below.

\begin{tabular}{|c|c|c|c|}
\hline $\begin{array}{c}\text { image } \\
\text { ry } \\
\text { noise }\end{array}$ & $\begin{array}{l}\text { imagery With } \\
\text { roar }\end{array}$ & $\begin{array}{l}\text { Noise } \\
\text { image }\end{array}$ & $\begin{array}{l}\text { imagery With } \\
\text { roar }\end{array}$ \\
\hline $15 \%$ & & $35 \%$ & \\
\hline $20 \%$ & & $40 \%$ & \\
\hline $25 \%$ & & $45 \%$ & \\
\hline
\end{tabular}




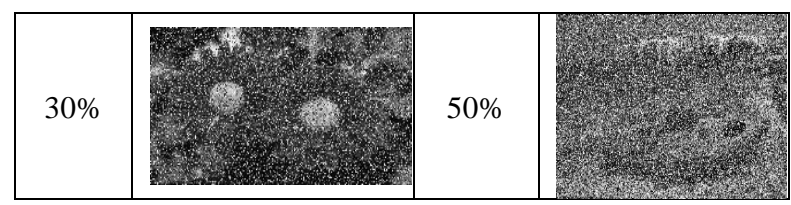

Figure 2.1: Data Image Noise $15 \%-50 \%$

\subsection{Stages Analysis}

Image by noise salt beforehand inputted and then will be the process of improving the quality of the image by a combination of noise reduction algorithm using SMF and the contrast enhancement algorithm using AFCEDP a different order depending on the 5 pieces of the image with the percentage of noise $15 \%$ - 50\% to get the best image of the by PSNR, Shannon Entropy (SE) and Contrast Improvement Evaluation (CIE).

The following stages of the process by the noise reduction and contrast enhancement using SMF AFCEDP:

\subsubsection{Spatial Algorithms stages Median Filter (SMF)}

Working procedure of the Spatial Median This filter can be described as shown in Figure 2.2 below:

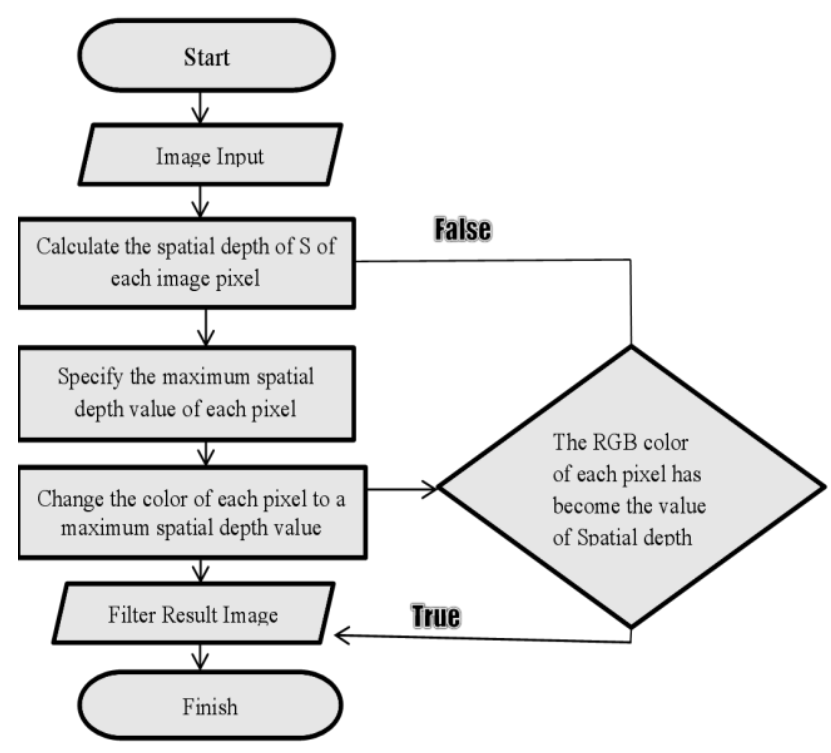

Figure 2.2: Flowchart Process Median Spatial Filter

a. Image input.

Suppose the input image size of $3 \times 3$ with the pixel color as table 2.1 below:

Table 2.1. Image Measuring 3 x 3

\begin{tabular}{|l|l|l|}
\hline 150 & 130 & 80 \\
\hline
\end{tabular}




\begin{tabular}{|c|c|c|}
\hline 88 & 90 & 38 \\
\hline 210 & 280 & 128 \\
\hline
\end{tabular}

b. Calculate the spatial depth of the $S$ of each image pixel.

For each pixel in the image, calculating the value of spatial depth. Suppose specified mask size is $2 \times 2$ so that $\mathrm{N}=4$ by using the following formula:

$$
S_{\text {depth }}\left(X, x_{1}, \ldots, x_{N}\right)=1-\frac{1}{N-1}\left\|\sum_{i=1}^{N} \frac{X-x_{i}}{\left\|X-x_{i}\right\|}\right\|
$$

c. Specify the maximum spatial depth value of each pixel.

After the calculated depth value of each pixel, then there will be the value of spatial depth max pixel selected from the Red value.

d. Change the color of each pixel to a maximum value of spatial depth.

If the red color of the first pixel is not a selected depth value, then replace the pixel values are selected to the first pixel.

e. The same thing will be repeated for all other pixel after the color RED all the pixels are processed.

f. If all the red has been processed, will be repeated back to GREEN and BLUE color of all pixels.

\subsubsection{Algorithm Process Stages AFCEDP}

The original image which is inputted in the form of a grayscale image. The image will be processed by algorithms Fuzzy Adaptive Contrast Enhancement algorithms with Preserving Details as for the process consists of four stages of processing are:

1. Determine the membership function of each entity.

2. Calculate the degree of membership and reference intensity values.

3. Defines 3 functions plateau and computing Clipping limit.

4. Doing Clipping and histogram equalization.

AFCEDP image algorithm results will be compared with the initial image by using Shannon Entropy and methods Improvement Evaluation Contrast this to show improved contrast and image detail preservation happens after repairs on the initial image, can be described as follows. Suppose the original image to be processed measuring 4 x 4 pixels, like the figure 2.3 below:

\begin{tabular}{|c|c|c|c|}
\hline 97 & 100 & 103 & 79 \\
\hline 108 & 86 & 97 & 103 \\
\hline 144 & 135 & 121 & 145 \\
\hline 169 & 85 & 153 & 50 \\
\hline
\end{tabular}

$$
p(k)=\frac{H(k)}{N}, \text { for } k=0,1, \ldots, L-1
$$

Figure 2.3. Example of First image

a. Membership Function Determination and Calculation of Degree of Membership

The initial image will be sought degree of membership for each pixel in the image. The technique used to divide the membership function for each pixel in the image below. 


$$
\begin{aligned}
& \mu_{\text {low }}(k)=\left\{\begin{array}{c}
0 \\
\frac{95-k}{20} \\
1
\end{array} \quad \mu \mu_{\text {tow }}(k)=\left\{\begin{array}{c}
0 \\
\frac{95-k}{20} \\
1
\end{array}\right.\right. \\
& \mu_{\operatorname{mid}}(k)=\left\{\begin{array}{c}
0 \\
\frac{k-75}{20} \\
1 \\
\frac{180-k}{20}
\end{array} \quad \mu_{\operatorname{mad}}(k)=\left\{\begin{array}{c}
0 \\
\frac{k-75}{20} \\
1 \\
\frac{180-k}{20}
\end{array}\right.\right. \\
& \mu_{\text {hisen }}(k)=\left\{\begin{array}{c}
0 \\
\frac{k-160}{20} \\
1
\end{array} \quad \mu_{k+k_{s h}}(k)=\left\{\begin{array}{c}
0 \\
k-160 \\
20 \\
1
\end{array}\right.\right.
\end{aligned}
$$

The end result of this process will form the membership function $\mu$ low $3, \mu$ mid, and $\mu$ high. Penggelempokkan process as follows.

1. Pixel-1 (Gray =97), then

lowfor pixel-1 is 0 (Gray> 95),

umidfor pixel-1 is 1 (Gray $\leq 95 \leq 160)$,

uhighfor pixel-1 is 0 (Gray $<160)$

2. The same calculation is done up to the last pixel or pixels-16

b. Analysis Intensity Reference Value Calculation

Reference intensity values obtained from the following formula.

$\lambda=($ Low_part $\times 43)+($ mid_part $\times 128)+(213 \times$ high_part $)$

c. Defining Analysis Function Three Plateau and Compute Clipping Limit

The third search function can use the following formula plateau.

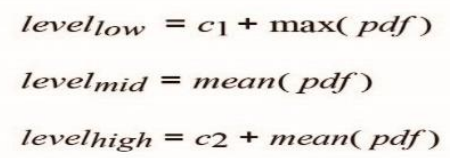

The third search function searches plateau starting from pdf (probability density function) which can use the formula.

Where the value of $\mathrm{H}(\mathrm{k})$ means many occurrences of pixels with gray-k can be seen in table 2.2.

\begin{tabular}{|c|c|c|c|}
\hline Value of gray & $\mathbf{P}(\mathbf{k})$ & Value of gray & $\mathbf{P}(\mathbf{k})$ \\
\hline 50 & 0.0625 & 108 & 0.0625 \\
\hline 79 & 0.0625 & 121 & 0.0625 \\
\hline 85 & 0.0625 & 135 & 0.0625 \\
\hline 86 & 0.0625 & 144 & 0.0625 \\
\hline 97 & 0125 & 145 & 0.0625 \\
\hline 100 & 0.0625 & 153 & 0.0625 \\
\hline 103 & 0125 & 169 & 0.0625 \\
\hline
\end{tabular}

Table 2.2 Calculation results $\mathrm{p}(\mathrm{k})$ 
C1 and C2 value used in the algorithm ranged between [-0015, -0005] and [0.005,0.007] sequentially. In this context the value of $\mathrm{c} 1$ and $\mathrm{c} 2$ used are -0.01 and 0.007 respectively. Thus, the value levellow, levelmid, levelhigh are:

levellow $=-0.01+0.125$ (the highest value of the pdf $)=0.115$

levelmid $=1 / 14$ (the average value of the $\mathrm{pdf})=0.0714$

levelhigh $=0,007+0.0714=0.0784$.

d. Clipping Analysis and Histogram Equalization

At this stage, carried out in the histogram equalization, to the value of $\mathrm{H}(\mathrm{k})$ and PDF are already received earlier in Table 3.1 and Table 3.2 respectively. The next step yajni pdf search new value using the following formula: $\quad n e w \_p(\mathrm{~K})=\min (\mathrm{p}(\mathrm{k}), \sigma(\mathrm{k}))$, for $\mathrm{k}=0, \mathrm{~L}-1$

1. Gray $=50$ $n e w \_p(50)=\operatorname{MIN}(0.0625,0.0714)=0.0625$

2. The same calculation is done until the final gray value in the image

Calculation continues to find the value of $\mathrm{c}(\mathrm{k})$ by using the formula:

Histogram smoothing is done using the following transformation function in order to obtain a new intensity value.

$$
n e w \_f(\mathrm{~K})=\mathrm{X} 0+(\mathrm{XL}-1-\mathrm{X} 0) \times(\mathrm{c}(\mathrm{k})-1 / 2 \mathrm{p}(\mathrm{k}))
$$

$\mathrm{X} 0$ and XL-1 refers to the lower limit and the upper limit of the histogram.Value X0 and XL-1 used in this example is 0 and 255 .

1. Gray value $=50$

$$
\begin{aligned}
n e w \_f(k) & =0+(255-0) *(0: 07-(0.5 * 0.07)) \\
& =8925(\text { rounded to } 8)
\end{aligned}
$$

New gray value will replace the previous gray value so as to form a new image that has been in the histogram equalization. Pictures of the final image can be seen in Figure 2.4.

\begin{tabular}{|c|c|c|c|}
\hline 82 & 100 & 121 & 26 \\
\hline 138 & 62 & 82 & 121 \\
\hline 192 & 174 & 156 & 210 \\
\hline 246 & 44 & 228 & 8 \\
\hline
\end{tabular}

Figure 2.4 Final image after histogram equalization

\subsection{Parameter Testing}

\section{a. Fidelity}

To determine the magnitude of the change and the quality of the image will be compared with the results of the initial image using the Mean Square Error (MSE) to determine differences in error and Peak Signal to Noise Ratio (PSNR) for measuring the quality of the image.

- Mean Square Error (MSE)

MSE is always inversely proportional to the value of PSNR. If the small MSE value it will generate a large PSNR value and vice versa. Usually the value of MSE is measured in percent (\%).

- Peak Signal To Noise Ratio (PSNR)

PSNR is usually measured in decibels (dB), the PSNR value falls below $30 \mathrm{~dB}$ indicates a fairly low image quality due to the low initial image quality due to noise and low contrast. For good quality and high value should PSNR of $40 \mathrm{~dB}$ or higher [7],

b. Details Image Information

The next test parameter is detail information of the image after increasing the contrast, using an algorithm Shannon Entropy Contrast Improvement and Evaluation robustness intends to determine the level of the amount of change in the image information. 


\section{Results}

In the process of improving the image with a combination algorithm, the results obtained from the combination of the best algorithm can be seen in Table 3.1:

Table 3.1 Percentage Noise image with contrast and image combination results

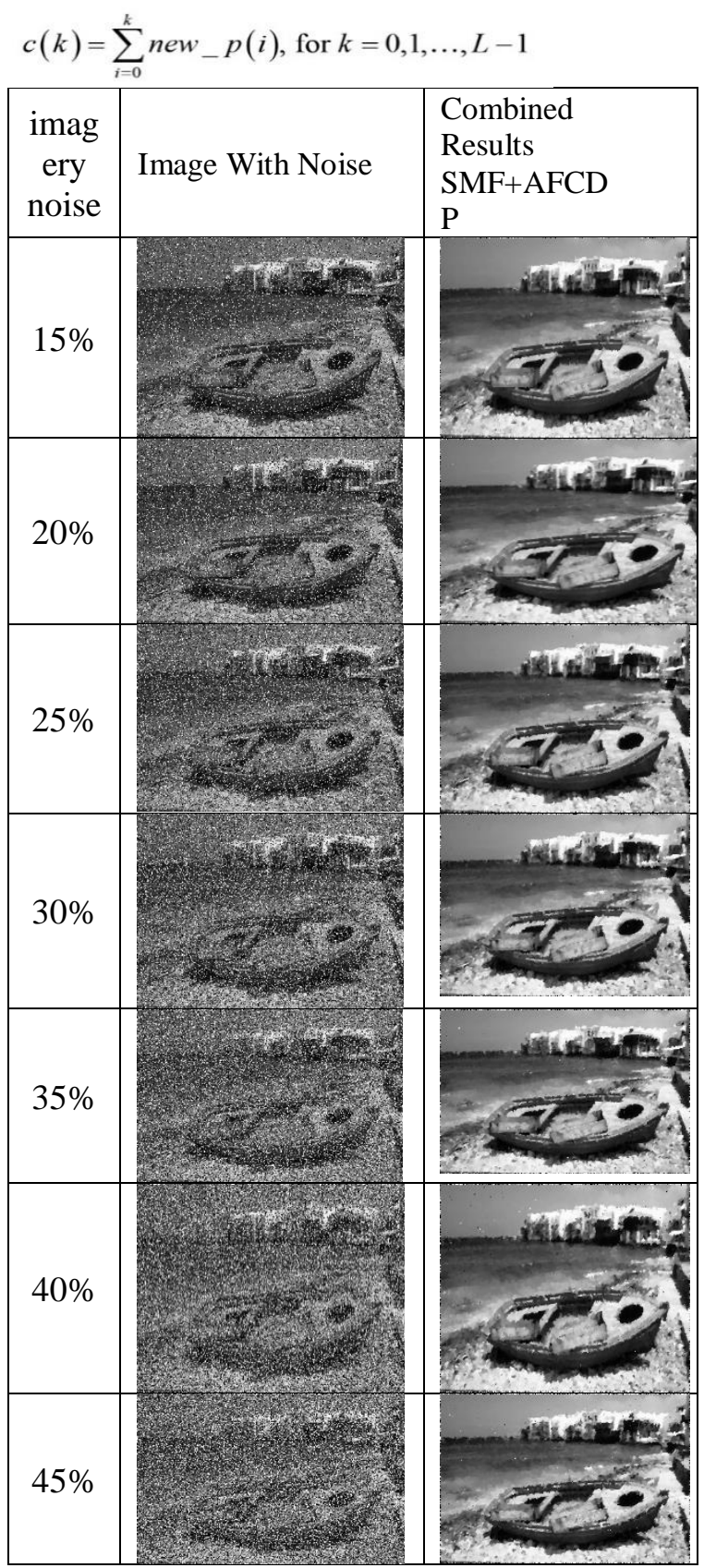




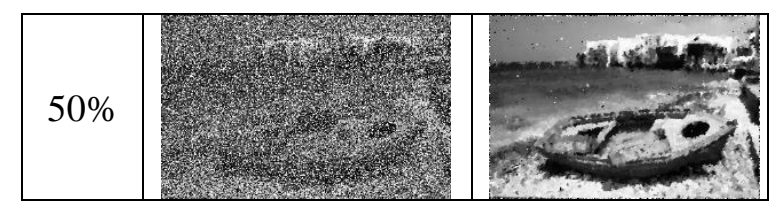

The results of each test performed image enhancement can didekripsikan best image enhancement test results of each persetasenoise $15 \%, 20 \%, 25 \%, 30 \%, 35.40 \%, 45 \%, 50 \%$ into a graph, in this case a combination of Median Spatial Filter (SMF) and Fuzzy Adaptive Contrast Enhancement Algorithm Preserving With Details (AFCEDP). Can be seen in Table 3.2 and Figure 3.1.

Table 4.11 Decryption of any image enhancement noise persetase

\begin{tabular}{|c|r|r|r|}
\hline Percentase Noise & MSE & \multicolumn{1}{|c|}{ PSNR } & SE \\
\hline $15 \%$ & 148.3689 & 26417 & 0.0387 \\
\hline $20 \%$ & 368.6323 & 22.4648 & 0.17495 \\
\hline $25 \%$ & 419.2204 & 21.9063 & 0.1855 \\
\hline $30 \%$ & 477652 & 21.3396 & 0.1819 \\
\hline $35 \%$ & 554604 & 20.6909 & 0213 \\
\hline $40 \%$ & 624639 & 20.1745 & 0.2177 \\
\hline $45 \%$ & 703832 & 19.6561 & 0.2181 \\
\hline $50 \%$ & 789317 & 19.1582 & 0.2457 \\
\hline
\end{tabular}

From the results of the above table can be drawn to suah chart as shown below 3.1

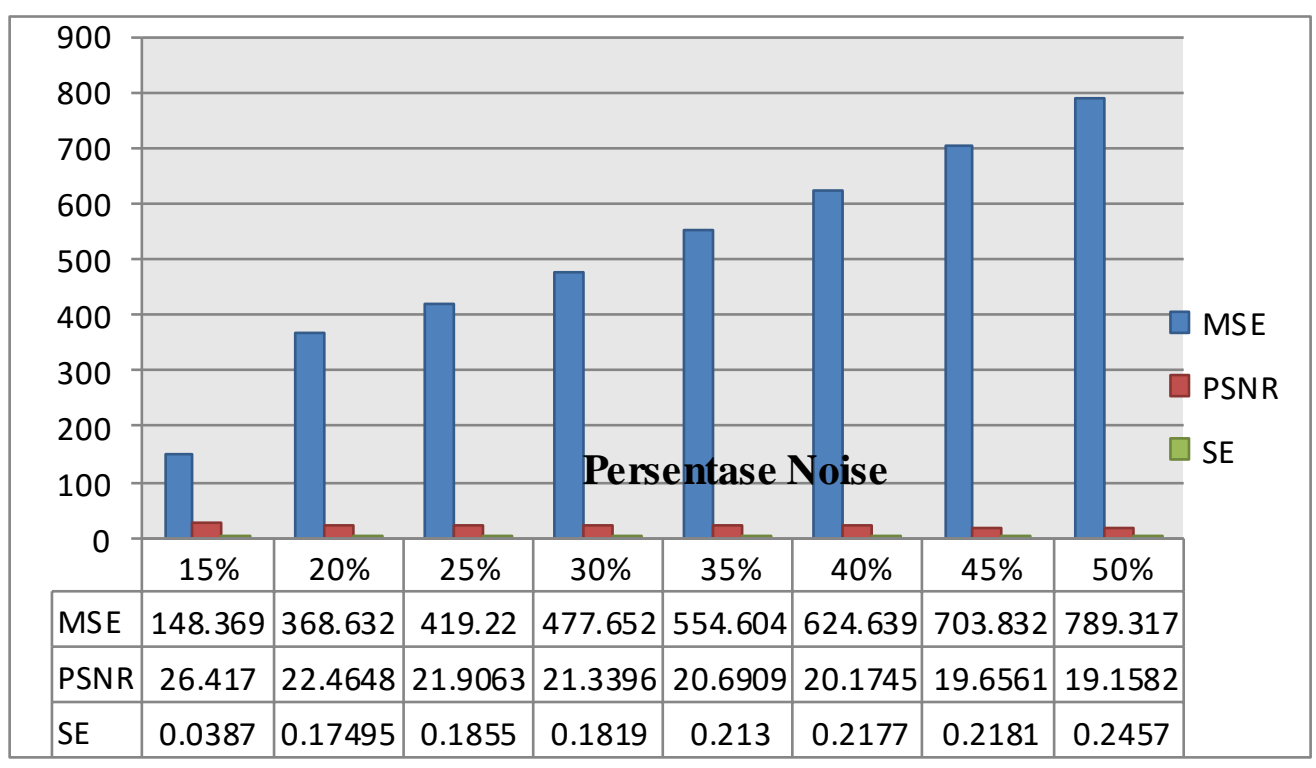

Figure 3.1 Chart Improved results from any percentages image noise. 


\section{Conclusion}

Based on an analysis of all test obtained a combination of increased maximum possible image quality by eliminating noise salt in advance using Spatial Median Filter (SMF) and then increase the contrast using Fuzzy Adaptive Contrast Enhancement Algorithm With Details Preserving (AFCEDP) than vice versa.

Highest image quality enhancement obtained at the noise percentage to $15 \%$ with $\mathrm{PSNR}=26417 \mathrm{~dB}$ and $\mathrm{SE}=0.0387$

This combination is becoming the new model in a manner that the image quality improvement do not breathing and able to improve the image quality of the results above 30 $\mathrm{db}$.

\section{References}

[1] T. Gebreyohannes and D. Kim, “Adaptive Noise Reduction Scheme for Salt and Pepper," Arxiv Prepr. arXiv1201.2050, 2012.

[2] E. J. Leavline, D. A. Antony, and G. Singh, "Salt and Pepper Noise Detection and Removal in Gray Scale Images : An Experimental Analysis,” vol. 6, no. 5, pp. 343-352, 2013.

[3] J. C. Church, Y. Chen, and S. V. Rice, "A Spatial Median Filter for noise removal in digital images," Conf. Proc. - IEEE SOUTHEASTCON, pp. 618-623, 2008.

[4] M. C. Mythili and D. V. Kavita, "Efficient Technique for Color Image Noise Reduction," Res. Bull. Jordan ACM, vol. 2, no. 3, pp. 41-44, 2011.

[5] A. K. Gupta, "Low Contrast Image Enhancement Technique By Using Fuzzy Method," vol. 4, no. 2 , pp. 518-526, 2016.

[6] J. R. Tang and N. A. M. Isa, "An Adaptive Fuzzy Contrast Enhancement Algorithm with Details Preserving," J. ICT Res. Appl., vol. 8, no. 2, pp. 126-140, 2014.

[7] L. Y. Por, D. Beh, T. F. Ang, and S. Y. Ong, "An enhanced mechanism for image steganography using sequential colour cycle Algorithm," Int. Arab J. Inf. Technol., vol. 10, no. $1,2013$. 